\title{
Prevalence of mutation of mismatch repair genes in young Egyptian patients with colorectal cancer
}

\author{
Ashraf A Mostafa, ${ }^{a} M D$; Samy Saad, ${ }^{a} M D ;$ Ahmed Alaa, ${ }^{a} M D$; \\ Ahmed Mohamed Ibrahim, ${ }^{a}$ MD; Maha Abd El-Wahab Mohamed, ${ }^{b}$ MD
}

\author{
a) Department of General Surgery, Ain Shams University, Cairo, Egypt. \\ b) Department of Pathology, Ain Shams University, Cairo, Egypt.
}

\begin{abstract}
Recent studies have demonstrated the cancer related role of genes that function in the repair of DNA damage. Inherited mutation of one of mismatch repair (MMR) genes results in hereditary non polyposis colorectal cancer (HNPCC). Even though HNPCC is the most common hereditary colon cancer, awareness of the disease among the public and health care providers is low and needs to be revived and established. The syndrome accounts for approximately 5\% of all colorectal cancers and for about 50\% of young cases. Five MMR genes have been implicated in HNPCC, but the majority of the mutations (95\%) are found in MLH1 and MSH2 genes. One hundred young patients ( $<50$ years) in Ain Shams University Hospitals with colorectal cancer were selected for this study from March 2003 to April 2007. The mean age was 41.5 years (range from 29-49 years). We tried to define two groups of patients, mutation group; that carried mutations in (MLH1 and /or MSH2) and the non mutation group. Moreover, we were concerned about the difference and similarity between both groups, as well as between our results and the other national and international studies of colorectal cancer (CRC) or HNPCC; regarding the demographic data, family history, anatomical data and pathological data. The surgical procedures were as follows: Anterior resection (39\%), right hemicolectomy (30\%), abdominoperineal resection (15\%), left hemicolectomy (6\%), transverse colectomy (3\%) and total colectomy (1\%). Thirty one patients of the studied group (31\%) showed mutation in either or both MLH1 and MSH2 (19 MLH1 mutation, 6 MSH2 and 6 both MLH1\&MSH2, with a predominance of MLH1 61.2\%).The mutation group compared to non mutation group had positive family history (Park criteria) (19\% versus 1.4\%), high prevalence of right sided tumors $51.6 \%$ versus $26 \%$ ), high incidence of poorly differentiated tumors(61.3\%), high mucinous tumors(45.2\%), but less lymph nodal involvement. Synchronous polyps were significantly higher in mutation group (19.4\%) and the only case with synchronous tumor was among the mutation group. In conclusion, the prevalence of mismatch repair gene in the studied young patients with CRCs is (31\%), which is lower than the same age group of Western CRCs patients (45\%).
\end{abstract}

\section{Introduction:}

Adenocarcinoma of the colon and rectum is a common cancer worldwide; it is the third most common site of the new cancer cases and deaths in the United States. In that country, the estimated incidence of new cases in 2002 was 148.300 with 56.600 deaths from the disease. The life risk of developing colorectal cancer in United States is 6\%; and over 90\% of cases occurring after the age of 50 years. ${ }^{1}$ Similar high incidence has been reported from Western European countries; Australia and
New Zealand. ${ }^{2}$ Lower rates have been reported in the third world and developing countries. ${ }^{3}$ Environmental factors, particularly dietary practices and physical inactivity are implicated in these striking geographic contrasts. ${ }^{4}$

The peak incidence for colorectal cancer is 60 to 70 years, fewer than $20 \%$ occur before the age of 50.5 In Egypt and other developing countries, there is a peculiar age distribution for colorectal cancer with almost one third of cases younger than 40 years. ${ }^{3}$ 
It is known that colorectal cancer can occur in hereditary, sporadic or familial forms. Hereditary forms are characterized by positive family history, young age of onset, and presence of other specific tumors and defects. Diagnosis of hereditary colorectal cancer is based on family history criteria, pathological criteria and recently, genetic criteria. It is now accepted that $80 \%$ of young onset colorectal cancer is hereditary. ${ }^{1}$ Recent studies have demonstrated the cancer-related role of genes that function in the repair of DNA damage. Hereditary nonpolyposis colorectal cancer (HNPCC), is characterized by early onset of colorectal cancer (fourth decade) without polyposis. ${ }^{6}$ It is inherited in an autosomal dominant manner, and arises from germline mutations in any of mismatch repair genes (MMR) with mutations of two of MMR (hMLH1 and hMSH2), accounting for more than $95 \%$ of mutations seen in HNPCC families. ${ }^{7-9}$

Colorectal cancers associated with HNPCC seem to be biologically different from colorectal cancers, where the tumors are more common in the right side of the colon, with about two-thirds of the cancer found proximal to the splenic flexure and synchronous and metachronous cancers are not uncommon. Patients who have inherited this condition are also at increased risk for other types of cancers particularly uterine $(40 \%)$, gastric $(20 \%)$, ovarian (10\%) and other rare cancers as biliary, small intestinal and brain cancers. 10

Several studies have also reported better survival rates in HNPCC-associated colorectal cancer than sporadic colorectal cancer when matched by cancer stage. ${ }^{11}$

Even though HNPCC is the most common hereditary colon cancer, accounting for up to $5 \%$ of all cases, awareness of the disease among the public and health care providers is low. One reason may be that HNPCC lacks the dramatic features of the less-common familial adenomatous polyposis (FAP). In this form of hereditary colon cancer, hundreds to thousands of polyps may be found carpeting the colon. On the other hand, the numbers of polyps in HNPCC patients is no greater than in general population, but progress to malignancy at younger age with $25 \%$ turning malignant at age of 50 and $80 \%$ at age of 60 years. ${ }^{12}$

Due to the peculiar young age distribution of colorectal cancer in Egyptian patients, the aim of this study was to define the prevalence of mutation of mismatch repair genes MLH1 $\&$ MSH2 in this group of patients with CRCs.

\section{Patients and methods:}

This study included 100 young ( $<50$ years) colorectal cancer patients. The patients were recruited from Ain Shams University Hospitals from March 2003 to April 2007. History with detailed family history was taken, routine ward procedures were done for every patient including, clinical examination and routine laboratory and radiological work up. Special investigations such as barium enema, CT, MRI, abdominal or rectal ultrasound were done according to the merits of each case. All specimens were subjected for routine histopathology as well as testing for mutation of mismatch repair genes MLH1 and MSH2. Exclusion criteria included, age 50 years or above, patients with colorectal cancer on top of familial polyposis or ulcerative colitis, because of the distinct genetic background of such diseases. Also patients with inoperable and irresectable tumors were excluded because of the unavailability of material for genetic study. All the patients were consented to participate in the study. The method of family history and testing for mismatch repair gene mutation were as follows:

\section{1) Family history:}

A detailed family history was taken to fill a pre-designed format with special concentration on colorectal cancer, other cancers elsewhere in the body and the cause of death in all first, second, third and possibly fourth degree relatives whenever possible. Reports of surgery done to family members were obtained whenever available. Patients were classified according to their family history into those fulfilling or not either Amsterdam Table(1) or Park criteria Table(2). Surgical procedures were tailored according to the site of the tumor. 


\section{2) Testing for mismatch repair gene mutation:}

\section{1- Collection and preparation of tissue samples:}

Two tissue sections were prepared from each resected specimen, one from the tumor (full thickness, 1 cubic $\mathrm{cm}$ ) and the other from the near by normal mucosa (mucosal block $1 \mathrm{~cm} \times 1 \mathrm{~cm}$ ) both were sent immediately to the pathology department to be preserved either frozen at the cryostat $(-21 \mathrm{Co})$ or as a paraffin block.

The original colonic or rectal specimens were then sent to routine histopathological examination to evaluate tumor type, grade, and degree of invasion, lymph node affection and staging.

\section{2- Immunohistochemical staining:} A- Reagents:

We used the Rabbit Immuno Cruz staining (Santa Cruz Biotech. Inc, USA) supplied with the following reagents:

1- Primary antibodies; MLH1 (code SC581) and MSH2 (code SC-494). These are purified rabbit polyclonal antibody raised against a peptide mapping at the amino terminus of MLH1and MSH2 of human origin, respectively.

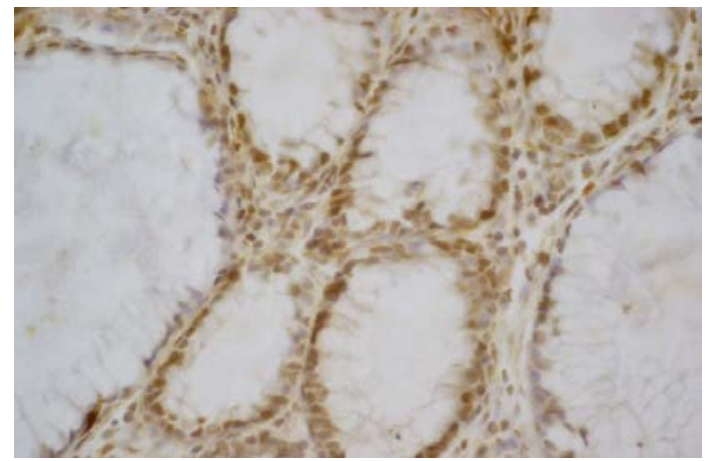

Figure (1): Positive control shows normal staining of the nuclei by MLHI (brown).

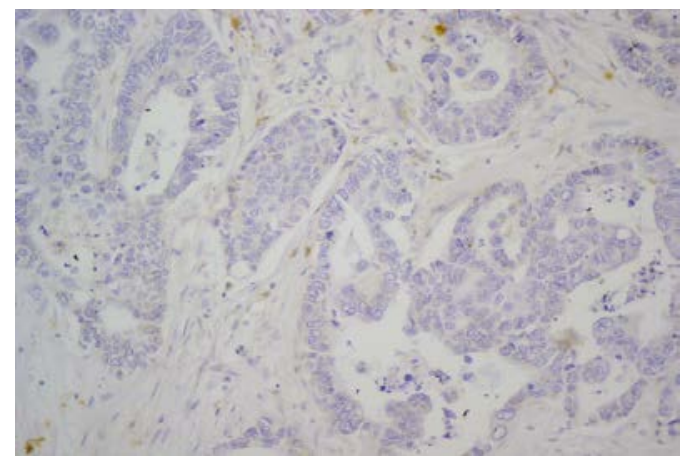

Figure (3): Adenocarcinoma with

negative staining of $\mathrm{MSH} 2$.
2- Universal Kit; (code SC-2051). This is a rabbit immuno cruz staining system. Each of these reagents is prediluted and ready to use for immunohistochemical staining of formalinfixed paraffin embedded tissue sections as well as frozen sections.

\section{B- Interpretation of stain (microscopic examination):}

Immunohistochemistry allowed detection of hMLH1 or hMSH2-MMR genes in colorectal tumor specimens Figure(1-6). Expression of the gene products resulted in positive staining of nuclei in proliferative cells of normal mucosa and tumor cells (brown color). Additionally, frequent cytoplasmic staining was observed.

Staining of nuclei in normal mucosa was used as internal positive control and compared to immunohistochemical staining of tumor cell nuclei Figure(1,2). If staining was missing in tumor cells Figure $(\mathbf{3 , 4 )}$ but positive immunodetection was found in normal mucosa, the sample was evaluated as lost expression of MLH1 or MSH2 protein.

\section{3) Statistical methodology:}

Analysis of data was done using SPSS (statistical program for social science).

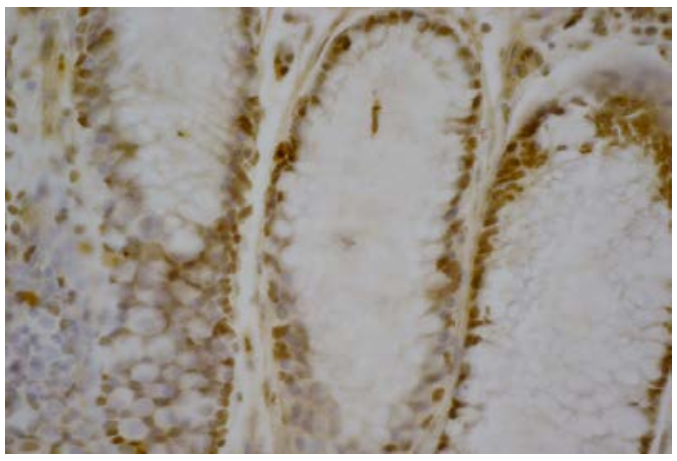

Figure (2): Positive control shows normal staining of the nuclei by MSH2.

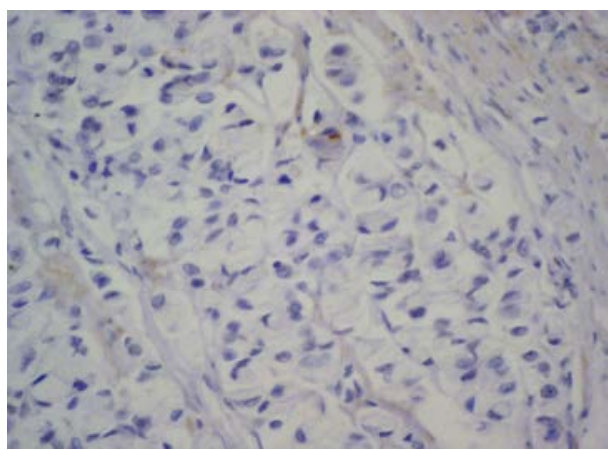

Figure (4): Adenocarcinoma with negative staining of MLHI. 


\section{Results:}

One hundred young Egyptian patients $(<50$ years, 62 males and 38 females) with colorectal cancer were subjected to this study. The mean age was 41.5 years (from 29 to 49 years).The main presenting symptoms were colicky pain (35\%), bleeding per rectum (31\%), intestinal obstruction (13\%), mucous discharge(11\%), and peritonitis(10\%).The anatomical distribution of the tumors and surgical procedures are listed in Table(3). Seventy two percent of the tumors were in the form of masses, $67 \%$ of the tumors had lymph node metastases, $51 \%$ were poorly differentiated, $25 \%$ were mucinous carcinomas, $10 \%$ had synchronous polyps in the resected specimens and one patient had synchronous cancer.

Table (1): Amsterdam criteria for diagnosis of hereditary nonpolyposis colorectal cancer.

\begin{tabular}{|c|l|}
\hline Name of criteria & \multicolumn{1}{c|}{ Specific criteria } \\
\hline 1- Amsterdam & $\begin{array}{l}\text { Three relatives with colorectal cancer, one of which is a first- } \\
\text { degree relative of the other two; colorectal cancer affecting more } \\
\text { than one generation; at least one colorectal cancer case diagnosed } \\
\text { before age 50 years. }\end{array}$ \\
\hline 2- Modified & $\begin{array}{l}\text { Two colorectal cancer cases in first-degree relatives in very small } \\
\text { families that cannot be expended further; colorectal cancer affecting } \\
\text { more than one generation; at least one colorectal cancer case } \\
\text { diagnosed before age 55 years. } \\
\text { Two first-degree relatives affected by colorectal cancer, plus a } \\
\text { third relative with an unusually early-onset neoplasm or endometrial } \\
\text { cancer. }\end{array}$ \\
\hline
\end{tabular}

* Fulfillment of all criteria listed in any paragraph in this section is sufficient. ${ }^{13}$

Table (2): Park criteria for diagnosis of hereditary nonpolyposis colorectal cancer.

\section{Criteria I}

At least one item from both Category 1 and Category 2 should be fulfilled. Category 1: Vertical transmission of colorectal cancer. At least two siblings affected with colorectal cancer in a family.

Category 2: Multiple colorectal tumors (including adenomatous polyp). At least one colorectal cancer diagnosed before the age of 50. Development of extracolonic cancer (endometrium, urinary tract, small intestine, stomach, hepatobiliary system, or ovary) in family members.

\section{Criteria II}

One colorectal cancer patients with at least one of the following:

1-Early age of onset ( $<40$ years).

2-Endometrial, urinary tract, or small intestine cancer in the index patient or a sibling (one aged $<50$ years).

3-Two siblings with other integral HNPCC extracolonic cancers (one aged $<50$ years).

\section{Revised criteria II*}

One colorectal cancer patient with at least one of the following:

1-Presence of synchronous or metachronous colorectal tumors in the patient, one of which occurred at less than age of 40 .

2-Endometrial, urinary tract, or small-intestine cancer in the patients or a first-degree relative (one aged $<50$ years).

3 -At least two first-degree relatives (one aged $<50$ years) with other extracolonic cancers (stomach, hepatobiliary system, ovary, or brain).

*The mutation detection rate using the original criteria II was low, so there was a definite need to revise criteria II. ${ }^{14}$ 
Table (3): Anatomical distribution of the tumor and surgical procedure among the studied cases.

\begin{tabular}{|c|c|c|c|}
\hline Anatomical distribution & No. & $\%$ & Surgical procedure \\
\hline Rectum & $39 \%$ & 39 & $\begin{array}{l}\text { Anterior resection }(24) \\
\text { Abdominoperineal resection(15) }\end{array}$ \\
\hline Rectosigmoid & $12 \%$ & 12 & Anterior resection (12) \\
\hline Sigmoid & $9 \%$ & 9 & $\begin{array}{l}\text { Pelvic colectomy (6) } \\
\text { Anterior resection (3) }\end{array}$ \\
\hline Left colon & $6 \%$ & 6 & Left hemicolectomy (6) \\
\hline \#Right colon & $34 \%$ & 34 & $\begin{array}{l}\text { Rt. hemicolectomy }(24) \\
\text { Extended Rt. hemicolectomy }(6) \\
\text { Transverse colectomy (3) } \\
\text { Total colectomy (1), synchronous cancer }\end{array}$ \\
\hline
\end{tabular}

\# Up to the splenic flexure.

None of the patients fulfilled the Amsterdam criteria. ${ }^{11}$ Seven patients $(7 \%)$ had positive family history of colorectal cancer; all were consistent with Park criteria for HNPCC (3 with Park I and 4 with Park II), three out of the seven patients had right sided tumors, while only one patient had synchronous polyp. Thirty one patients of the studied group (31\%) showed mutation in either or both MLH1 and MSH2 (19 patients with MLH1 mutation, 6 patients with MSH2 and 6 patients showed both MLH1\&MSH2). Four (67\%) of those with mutation and positive family history $(n=6)$ harbored MLH1 mutation while in the remaining two (33\%) MSH2 mutation was disclosed.

Seventy two percent of the tumors were in the form of masses, $67 \%$ of the tumors had lymph node metastases, $51 \%$ were poorly differentiated, $25 \%$ were mucinous carcinomas, $10 \%$ had synchronous polyps in the resected specimens and one patient had synchronous cancer.

There was no significant difference as regard age and sex distribution or clinical presentation among the mutation subgroups. On the other hand six patients $(19.4 \%)$ of the mutation group had positive family history of colorectal cancer fulfilling either Park I OR II criteria, whereas one patient (1.4\%) of the non mutation group had positive family history of colorectal cancer that fulfilled Park II criteria. None of the patients or their family members had history of extra colonic malignancies.

Sixteen patients $(51.6 \%)$ of the mutation group had right sided tumors. This was statistically significant $(\mathrm{p}<0.05)$ when compared to the right sided tumors in the non mutation group (26\%). There was no significant difference between mutation and non mutation group regarding the site of the tumor elsewhere in the colon or rectum Table(4).

Table (4): Comparison between the studied groups as regard anatomical distribution of the tumor.

\begin{tabular}{|l|c|c|c|}
\hline $\begin{array}{l}\text { Anatomical } \\
\text { distribution }\end{array}$ & $\begin{array}{c}\text { Mutation group } \\
\mathbf{n = 3 1}\end{array}$ & $\begin{array}{c}\text { No mutation } \\
\mathbf{n = 6 9}\end{array}$ & $\mathbf{P}$ \\
\hline Rectum & $9(29 \%)$ & $30(43.5 \%)$ & $>0.05$ \\
\hline Rectosigmoid & $4(12.9 \%)$ & $9(13 \%)$ & $>0.05$ \\
\hline Sigmoid & 0 & $9(13 \%)$ & $>0.05$ \\
\hline Left colon & $3(9.7 \%)$ & $3(4.3 \%)$ & $>0.05$ \\
\hline Right colon & $16(51.6 \%)$ & $18(26 \%)$ & $<0.05$ \\
\hline
\end{tabular}


There was significant difference between mutation group and non mutation groups regarding the presence of poorly differentiation, mucinous adenocarcinoma and the presence of synchronous polyps or cancer $(\mathrm{p}<0.05)$, all being more frequent among the mutation group. On the other hand, lymph node metastases were significant among non mutation group $(\mathrm{p}<0.05)$ Table(5).

Table (5): Comparison between the studied groups as regard pathological features.

\begin{tabular}{|l|c|c|}
\hline Pathological features & $\begin{array}{c}\text { Mutation group } \\
\mathbf{n = 3 1}\end{array}$ & $\begin{array}{c}\text { No mutation } \\
\mathbf{n = 6 9}\end{array}$ \\
\hline Poorly differentiated & $19(61.3 \%)$ & $32(46.4 \%)$ \\
\hline Mucin positive & $14(45.2 \%)$ & $11(15.9 \%)$ \\
\hline L.N. affection & $19(61.3 \%)$ & $48(69.6 \%)$ \\
\hline Synchronous polyps & $6(19.4 \%)$ & $4(5.8 \%)$ \\
\hline Synchronous CRC & $1(3.2 \%)$ & 0 \\
\hline
\end{tabular}

Among the mutation group, 8 patients (42.1\%) with mutation in MLH1 had poorly differentiated tumors, compared to 5 patients $(83 \%)$ in $\mathrm{MSH} 2$ mutation subgroup. Also in patients MLH1 mutation subgroups, there was less lymph node affection and less synchronous polyps found in this subgroup. Otherwise there was no significant difference between patients with mutation in MLH1 and MSH2 in any of the other compared parameters (gender, family history, anatomical site and presence of mucin Table(6).

Table (6): Comparison between the mutation subgroups as regard demographic, anatomical and histopathological data.

\begin{tabular}{|l|c|c|c|}
\hline Variables & $\begin{array}{c}\text { Subgroup A } \\
\text { MLH1 mutation } \\
\mathbf{n}=\mathbf{1 9}\end{array}$ & $\begin{array}{c}\text { Subgroup B } \\
\text { MSH2 mutation } \\
\mathbf{n}=\mathbf{6}\end{array}$ & P \\
\hline $\begin{array}{l}\text { Gender } \\
\text { Males } \\
\text { Females }\end{array}$ & $\begin{array}{c}11(58 \%) \\
8(42 \%)\end{array}$ & $\begin{array}{c}2(33.3 \%) \\
4(66.7 \%)\end{array}$ & $>0.05$ \\
\hline Family history \\
positive (Park criteria)
\end{tabular}$\quad$\begin{tabular}{c}
$2(33.3 \%)$ \\
\hline Anatomical site \\
Rt. colon Rectum
\end{tabular}




\section{Discussion:}

More than 30\% of CRC patients in Egypt are younger than 40 years. ${ }^{3}$ Therefore, we tried to define the prevalence of mutation of MLH1 \& MSH2 among young CRC patients and its contribution to the high prevalence of CRC in this age group.

As we proceeded in this study we tried to define two groups of patients; mutation group, that carried mutations in (MLH1 and /or MSH2) and the non mutation group. We considered the mutation group as HNPCC group; defined by having a mutation in either genes. ${ }^{15}$ Moreover, we were concerned about the difference and similarity between both groups, as well as between our results and the other Egyptian and international studies of CRC or HNPCC; as regard to demographic data, family history, anatomical data and pathological data.

Though males in our series represented $67 \%$ of the non mutation group, there was an even distribution between males and females among the mutation group. The difference between the two groups was, however, statistically insignificant $(\mathrm{P}>0.05)$. This result is similar to those reported by Abou Zeid and his associates in $2002^{3} \&$ Soliman and his associates in $2001^{16}$ in two separate studies on Egyption CRC and agrees also with the results of Liu and his associates in $2004{ }^{17}$ on Chinese patients with HNPCC with a male to female ratio of 1.2:1.

The main stay of the diagnosis of HNPCC is a detailed family history, although Salovara and his associates in $2000^{18}$ reported $20 \%$ of newly discovered cases to be due to spontaneous germ line mutations. None of our 100 patients fulfilled Amsterdam criteria, while 7(7\%) were, either Park I criteria (3 cases) or Park II criteria (4cases) positive. In the mutation group, $19.4 \%$ of the patients fulfilled Park I\&II criteria, on the other hand only one patient $(1.4 \%)$ of the non-mutation group fulfilled Park II criteria. This difference was found to be statistically significant $(\mathrm{P}<0.05)$. Therefore we speculate that the positivity of the family history carries a great significance in HNPCC patients. Even though more than $80 \%$ of the mutation group had negative family history, more than $85 \%$ of patients with positive family history belong to the mutation group. This means that absence of family history doesn't exclude or even reduce the incidence of occurrence of MMR genes mutation among young Egyptian CRC patients. This low prevalence of positive family history among Egyptian patients was noticed in the work of both Abou Zeid and his associates in $2002^{3}$ \& Soliman and his associates in $2001^{16}$ where they reported a prevalence of $(4.5 \%)$ and $(1.7 \%)$, respectively. The relatively higher results in this report compared to the previously mentioned studies, may be attributed to the age selection criterion in our study which raised the number of HNPCC cases among our patients i.e. "selection bias".

The low prevalence of the positive family history among our HNPCC patients was also observed in both Chinese HNPCC 17 and Japanese HNPCC patients. ${ }^{18}$ Furthermore, it was reported recently by western authors. ${ }^{19,20}$ This low prevalence of positive family history could be attributed to de-novo HNPCC (spontaneous germ line mutation) ${ }^{21}$ or it could reflect presence of exogenous environmental factor that predisposed to germ line mutation. ${ }^{22}$

We found a high prevalence of MLH1 mutations compared to MSH2 among patients with positive family history in the mutation group (66.7\% with MLH1 mutations versus 33.3 with $\mathrm{MSH} 2$ mutations). Interestingly Park and his associates in 199714 noticed that MLH1 mutations among Park criteria positive patients represented also $66.7 \%$ of the totally observed 24 mutations and the remainder showed MSH2 mutations.

A preferential proximal location of HNPCC has been reported in many studies and up to $70 \%$ of HNPCC were right sided among both Western patients ${ }^{23}$ and Chinese patients. ${ }^{17}$ Thirty four percent of the total tumors in this study were right sided (starting from the cecum up to the splenic flexure) with a significant $(\mathrm{P}<0.05)$ high prevalence of right sided adenocarcinoma among the mutation group $(51.6 \%)$ compared to non-mutation group (26\%). This high prevalence of right sided adenocarcinoma among the mutation group was attributed to the unique nature of HNPCC. ${ }^{24}$ 
Compared to Western CRC we found lower prevalence of right sided tumors $(51.6 \%)$, with higher prevalence of rectal carcinoma (29\%) among our HNPCC patients. This was similarly reported by Soliman and his associates in 2001,16 with more than $50 \%$ of the Egyptian CRC presented at the rectum. They suggested that this peculiar distribution could be attributed to the presence of exogenous factor responsible for carcinogenesis.

Fifty one percent of the totally examined tumors were poorly differentiated. This percent increased among the mutation group $(61.3 \%)$ compared to the non-mutation group (46.3\%) $(\mathrm{P}<0.05)$. This incidence is considered higher than that reported by Western authors. ${ }^{25}$ This may be attributed to the high prevalence of poorly differentiated adenocarcinoma among Egyptian patients where "more than 50\% of Egyptian CRC was poorly differentiated". 16

Again, $25 \%$ of the total examined tumors were mucinous. This high prevalence was reflected on the mutation group, where mucinous tumors were $(45.2 \%)$ compared to $(15.9 \%)$ in non-mutation group. This significant high prevalence of mucinous adenocarcinoma among the mutation group $(\mathrm{P}<0.05)$ may be part of the general high prevalence of mucinous adenocarcinoma among Egyptian CRC. ${ }^{3}$

Lymph nodal involvement was found in $67 \%$ of the total examined specimens. The high prevalence of lymph nodal involvement in this study compared to western CRC patients could be related to the late presentation and diagnosis of CRC among our patients or due to the aggressiveness of the tumors.

Furthermore, we found that nodal involvement was lower among the mutation group $(61.3 \%)$ compared to non-mutation group $(69.6 \%)$. This result contradicts with that of Redston and his associates in $2001^{25}$ who noticed a high prevalence of lymph nodal involvement among HNPCC than sporadic CRC Western cases.

Synchronous cancers were found in up to $18 \%$ of Western HNPCC patients. ${ }^{26}$ We had only one patient $(1 \%)$ of the total examined patients with synchronous tumor, and it was among the mutation group (3.2\%). Though this result could not be considered significant, it was nearly similar to that of Liu and his associates in 200417 reports on Chinese HNPCC (3.1\% with synchronous tumors), and to Abou Zeid and his associates in $2002^{3}$ on Egyptian CRC patients (4.5\% with synchronous tumors).

On the other hand the presence of synchronous polyps was noticed in $10 \%$ of the total examined specimens in the present work. This was significantly higher $(\mathrm{P}<0.05)$ among the mutation group (19.4\%) compared to nonmutation group $(5.8 \%)$. Our result was higher than that of Abou Zeid and his associates in $2002^{3}$ who stated that only $5.6 \%$ of Egyptian CRC had synchronous polyps. This may be due to the age selection criterion in our study.

Interestingly, the incidence of synchronous polyps in the mutation group was $19.4 \%$. The incidence of synchronous polyps in the Western literatures is not consistent as it is reported by Stormorken and his associates in $2005^{24}$ to be "30\% of HNPCC cases" whereas Koch and his associates in $2005^{23}$ observed in their work that $14 \%$ of the mutation group has synchronous polyps.

A clear predominance of MLH1 mutation (61\%) over MSH2 mutation (31.5\%) was observed in our study. This predominance of MLH1 was reported in many western studies $23,27,28$ with similar results (70\% MLH1 and $50 \% \mathrm{MSH} 2$ ) reported by the latter authors.

Except for the significantly higher MLH1 mutation among right CRC, Kruschewski and his associates in $2002^{27}$ found no significant difference between MLH1 and MSH2 subgroups among western HNPCC patients as regard tumor stage, lymph nodal involvement and tumor differentiation. We noticed that tumors of MLH1 mutation subgroup was significantly less poorly differentiated $(\mathrm{P}<0.05)$, had synchronous polyp $(\mathrm{P}<0.05)$ and with less lymph nodal involvement $(\mathrm{P}<0.01)$. On the other hand, there was no significant difference in right side predominance in both MLH1\& MSH2 mutation subgroup.

Thirty one percent of the total examined cases had mutations of MLH1 and/or MSH2. This, finding is discordant with Thibodeau and his associates in $1996^{29}$ and Kruschewski and his associates in $2002^{27}$ who found mutations in $28 \%$ and $12 \%$ of Western CRC patients, respectively. Their results, however, did not 
consider the age as a selection criterion. Nonetheless and finally, Stormorken and his associates in $2005^{24}$ noticed that $45 \%$ of young western CRC patients (similar to our age group) had mutations of MLH1 and /or MSH2.

\section{Conclusion:}

The prevalence of Mismatch repair gene in this work (31\%) is lower than the same age group of Western CRCs patients (45\%). This lower prevalence cannot solely explain what has been reported by Abou Zeid and his associates in $2002^{3}$ that more than $30 \%$ of CRC patients in Egypt were younger than 40 years. We believe, nevertheless, that it may pave the road for further researches to explain this peculiar phenomenon.

\section{References:}

1- Nease D, Stoffel E, Turgeon K, Ruffin M: Colorectal cancer screening clinics in family practice. Dis Colon Rectum 2004; 31(6): 43-51.

2- Parkin D, Pisani P, ferlay J: Estimates of the world incidence of 18 major cancers in 1985. Int J cancer 1993; 54: 495-606.

3- Abou Zeid A, Khafagy W, Marzouk D, et al: Colorectal cancer in Egypt. Dis Colon Rectum 2002; 45(9): 1255-1260.

4- Ponz De Leon M, Benatti P, Borghi F, et al: Etiology of colorectal cancer and relevance of monogenic inheritance. Gut 2004; 53: 115-122.

5- Pensotti V, Radice P, Prescuiuttini S, et al: Mean age of onset in HNPCC families correlate with the presence of mutations in DNA mismatch repair genes. J Genes Chromosomes Cancer 1997; 19: 135-142.

6- Greenlee R, Hill M, Murray T, et al: Cancer statistics. CA Cancer J Clin 2001; 15-36.

7- Lynch H, De La Chapell A: Genetic susceptibility to non polyposis colorectal cancer. J Med Genet 1999; 36: 801-818.

8- Chung D: The genetic basis of colorectal cancer: Insight into critical pathways of tumorogenesis. Gastroenterology 2000; 119: 854-865.

9- Scartozzi M, Bianchi F, Rosati S, et al: Mutations of hMLH1 and hMSH2 in patients with suspected HNPCC. J Clin Oncol 2002; 20: 1203-1208.
10-Dunlop M, Farrigton S, Murray T, et al: Cancer risk associated with germline DNA mismatch repair gene mutation. Hum Mol Genet 1997; 6: 105-110.

11-Vasen H,Waston P, Meckline J, et al: New clinical criteria of hereditary non polyposis colorectal cancer proposed by ICC on HNPCC. Gastroenterology 1999; 116: 1453-1456.

12-Marra G, Boland C: Hereditary non polyposis colorectal cancer: The syndrome, the genes and historical prespective. J Natl Cancer Inst 1995; 87: 1114-1125.

13-Jarvinen H, Aarnio M: Surveillance of mutation carriers of DNA mismatch repair genes. Ann Chir et Gyn 2000; 89: 207-210.

14-Park J, Vasen H, Murray T, Park K, et al: Suspected HNPCC. Cancer Res 1997; 42: 710-721.

15-Mitchell R, Farrington S, Dunlop M, Campell H: Mismatch repair genes MLH1 and MSH2 and colorectal cancer. AM J Epidemiology 2002; 156: 889-902.

16-Soliman A, Bondy M, El-Badawy S: Contrasting molecular pathology of colorectal carcinoma in Egyptian and Western patients. British J Cancer 2001; 85: 1037-1046.

17-Liu S, Zhao B, Wang Z, et al: Clinical features and mismatch repair gene mutation screening in Chinese patients with HNPCC. World J Gastroenterol 2004; 10: 2647 2651.

18-Salovara R, Loukola A, Kristo P, et al: Population based molecular detection of HNPCC. J Clin Oncol 2000; 18: 2193 2200.

19-Grady W: Molecular basis for subdividing hereditary colon cancer. Gut 2005; 54: 1676-1678.

20-Tomita N, Fukunga M, Okamura S, et al: The novel germline mutation of MLH1and $\mathrm{MSH} 2$ in cases of suspecting HNPCC in patients with no family history of cancer. Jap J Clin Oncol 2004; 34: 556-560.

21-Durno C, Aronson M, Bepat B, et al: Family history and molecular features of children, adolescence and young adults with colorectal carcinoma. Gut 2005; 54: 1146 1150 . 
22-Mahdavini M, Bishehsari R, Stoffel E, et al: Family history of colorectal cancer in Iran. BMC Cancer 2005; 5: 112-123.

23-Koch Y, Vogelsang H, Kopp R, et al: HNPCC: Clinical and molecular evidence for a new entity of hereditary colorectal cancer. Gut 2005; 54: 1733-1740.

24-Stormorken A, Bowtiz I, Noven T, et al: Immunohistochemistry identifies carriers of mismatch repair genes defects causing HNPCC. Clin Oncol J 2005; 23: 47054712.

25-Redston M: Carcinogenesis in GI tract: From morphology to genetics and back again. Pathology 2001; 14: 236-245.

26-Box J, Rodriguez M, Weber T, et al: Clinical implications of multiple colorectal carcinomas in HNPCC. Dis colon Rectum 1999; 42(6): 717-721.
27-Kruschewski M, Noske A, Hier J, et al: Reduced impression of mismatch repair genes MLH1 and MSH2 in patients with sporadic colorectal cancer related to their prognosis? Clinical and Experimental Metastasis J 2002; 19: 781-787. 28-Ruszkiewicz A, Bennett G, Moor J, et al: Correlation of mismatch repair genes immunohistochemistry and microsatellite instability status in HNPCC associated tumors. Pathology 2002; 34: 541-547. 29-Thibodeau S, French A, Roche P, et al: Altered expression of hMSH2 and hMLH1 in tumors with microsatelite instability and genetic alterations in mismatch repair genes. Cancer Res 1996; 56: 4836-4840. 